\title{
Quarks, Electrons, and Atoms in Closely Related Universes
}

\author{
Craig J. Hogan \\ Astronomy and Physics Departments, \\ University of Washington, Seattle, Washington 98195-1580
}

\begin{abstract}
In a model where a multiverse wavefunction explores a multitude of vacua with different symmetries and parameters, properties of universes closely related to ours can be understood by examining the consequences of small departures of physical parameters from their observed values. The masses of the light fermions that make up the stable matter of which we are made - the up and down quarks, and the electron - have values in a narrow window that both allows a variety of nuclei other than protons to exist, and at the same time allows atoms with stable shells of electrons that are not devoured by their nuclei. These fundamental parameters of the Standard Model are good candidates for quantities whose values are determined through selection effects within a multiverse, since a living world of molecules needs stable nuclei other than just protons and neutrons. If the fermion masses are fixed by brane condensation or compactification of extra dimensions, there may be observable fossils of the branching event, such as a gravitational wave background.
\end{abstract}




\section{INTRODUCTION}

We know that nature is governed by mathematics and symmetries. Not very long ago, it was an article of faith among most physicists that everything about physics would eventually be explained in terms of fundamental symmetries - that nothing in the makeup of physical laws is accidental, that nature ultimately has no choices, and that all the properties of particles and fields are fixed by pure math.

In the thirty years since modern anthropic reasoning was introduced into cosmology [1, 2], the competing idea that anthropic selection might have an indispensable role in fundamental physical theory has gradually become, if not universally accepted, at least mainstream. There are now concrete physical models for realizing anthropic selection in nature. Cosmology has provided not only a concrete mechanism (inflation) for manufacturing multiple universes, but also a new phenomenon (Dark Energy) whose value is most often explained by invoking anthropic explanations. String theory has uncovered a framework by which many different symmetries and parameters for fields can be realized in the low-energy, $3+1$ dimensional universe, depending on the topology and size of the manifold of the other seven, truly fundamental dimensions, and on the configurations of p-branes within it, especially the local environment of the 3-brane on which our own Standard Model fields live. The numbers of locally metastable configurations of manifold and branes, and therefore the number of options for low energy physics, are estimated to be so large that for all practical purposes, there is a continuum of choices for fundamental parameters that we observe [3, 4 , [5].

Of course, the details of how this works in the real world are still sketchy. Cosmology unfolds in a series of phase transitions and symmetry breakings. For example, it is now part of standard inflation that the quantum wavefunction of the universe branches early into various options for the zero-point fluctuations of the inflaton field, different branches of which correspond to different distributions of galaxies. String theory opens up a scenario in which the multiverse wavefunction may also branch very early into a variety of whole universes, each of which has different physics. If things happen this way, it is natural for us to find ourselves in a branch with physics remarkably well suited to make the stuff of which we are made.

It then makes sense to ask new questions about the world: how would things change if this or that aspect of physics were changed? If a small change in a certain parameter 
changes the world a great deal in a way that matters to our presence here, that is a clue that that particular parameter is fixed by selection rather than by symmetry. The following arguments along these lines are elaborated more fully in ref. [6].

Now we may be faced where a situation where some seemingly fundamental features of physics might not ever be derived from first principles. Even the particular gauge group in our Grand Unified Theory (that is, the the one in our branch of the wavefunction) might be only one group selected out of many options provided by the Theory of Everything. We may have to adjust our scientific style to this larger physical reality, which forces cosmology and fundamental physics into a new relationship. For example, although we can't look inside the other universes of the multiverse ensemble and can't predict the branching outcome from first principles, cosmological experiments now under development might reveal relict gravitational waves from the same symmetry breaking that fixed the parameters.

\section{CHANGING STANDARD MODEL PARAMETERS}

Evaluating changes in the world in response to changes in the fundamental physics is actually a difficult program to carry out. For the most fundamental theory we have, the Standard Model, the connection of many of its parameters with generally observable phenomena can only be roughly estimated. First-principles calculations of the behavior of systems such as nuclei and molecules are possible only for the simplest examples.

The traditional minimal Standard Model has 19 "adjustable" parameters [7, 8]: Yukawa coefficients fixing the masses of the six quark and three lepton flavors $(u, d, c, s, t, b, e, \mu, \tau)$, the Higgs mass and vacuum expectation value $v$ (which multiplies the Yukawa coefficients to determine the fermion masses), three angles and one phase of the CKM (Cabibbo-KobayashiMaskawa) matrix (which mixes quark weak- and strong-interaction eigenstates), a phase for the quantum chromodynamic (QCD) vacuum, and three coupling constants $g_{1}, g_{2}, g_{3}$ of the gauge group, $U(1) \times S U(2) \times S U(3)$. We now know experimentally that the neutrinos are not massless, so there are at least seven more parameters to characterize their behavior (three masses and another four CKM matrix elements). Thus 26 parameters, plus Newton's constant $G$ and the cosmological constant $\Lambda$ of General Relativity, are enough to describe the behavior of all the observed particles in all experiments, except those related to new

Dark Matter particles. If in addition the Standard Model is extended by supersymmetry, 
the number of parameters exceeds 100.

Imagine that you are sitting at a control panel of the universe. It has a few dozen knobsone for each of the parameters. Suppose you start twiddling the knobs. For all but a few of the knobs, you find nothing changes very much; the mass of the top quark for example (that is, its Yukawa coupling coefficient in the Standard Model equations) has little direct effect on everyday stuff. Which knobs matter for the stuff we care about most - atoms and molecules?

Some knobs are clearly important, but their exact value does not seem too critical. The fine structure constant $\alpha$ for example controls the sizes of all the atoms and molecules, scaling like the Bohr radius $\left(\alpha m_{e}\right)^{-1}$. If you twiddle this knob, natural phenomena dominated by this physics - which includes all of familiar chemistry and biology - grow or shrink in size. On the other hand they all grow or shrink by almost the same fractional amount so the structural effect of changes is hard to notice; the miraculous fit of base pairs into the DNA double helix would still work pretty well. There are however subtle changes in structural relationships and molecular reaction rates. Your complicated biochemistry probably would not survive a sudden big change in $\alpha$, but if you turn the knob slowly enough, living things probably adapt to the changing physics. Simulations of cellular reaction networks show that their behavior is remarkably robust with respect to changes in reaction rates, and mostly depend on network topology 9$]$.

It turns out that a few of the knobs have a particularly large qualitative effect with a very small amount of twiddling. Three knobs stand out for their particularly conspicuous effects: the Yukawa coefficients controlling the masses of the electron, the up-quark, and the down-quark. They are the light fermions that dominate the composition and behavior of atoms and molecules. Changing them by even a small fractional amount has a devastating effect on whether molecules can exist at all. The most dramatic sensitivity of the world on their values seems to be in the physics of atomic nuclei.

\section{EFFECTS OF CHANGING $u, d, e$ MASSES ON ATOMS AND NUCLEI}

The light fermion masses are all very small (less than one percent) compared with the mass of even a single proton. (Protons and neutrons, which comprise the bulk of the mass of ordinary matter, ironically have a mass dominated not by the "real mass" of their matter 
particles, the constituent quarks, but almost entirely by the kinetic energies of the quarks and the massless gluons mediating the color forces.) However, the light fermion masses are critical because they determine the energy thresholds for reactions that control the stability of nucleons.

In the three dimensional parameter space formed by these masses, the most reliable phenomemological statements can be made about changes within the two dimensional surface defined by holding the sum of $u$ and $d$ masses constant. (That is because many complicated features of nuclear physics remain constant if the pion mass, which is proportional to $\left(m_{u}+m_{d}\right)^{1 / 2}$, is constant). In this plane, some properties of worlds with different values of the masses are summarized in figures 1 and 2, the latter taken from ref. [6]. The figures also show a constraint from a particular $\mathrm{SO}(10)$ grand unified scenario, to illustrate that likely unification schemes probably do not leave all these parameters independent- at least one relationship between them is likely fixed by unification symmetry.

In the lower part of figure 1, towards larger up-quark mass, there are "Neutron Worlds". As one dials knobs in this direction, a threshold is soon crossed where it is energetically favorable for the electron in a hydrogen atom to join with its proton to make a neutron. If you turn it farther, even a free proton (without any nearby electron) spontaneously decays into a neutron.

In the upper part of the figure, there are "Proton Worlds". Moving up from our world, a threshold is soon crossed where a deuteron in a plasma is no longer energetically favored over a pair of protons. If you go farther, even an isolated deuteron spontaneously decays into a pair of protons.

In the neutron world, there are nuclei, but not atoms with electrons around them, so chemistry does not happen. In the proton world, there are hydrogen atoms, but that is the only kind of atom, because the other nuclei do not form or are not stable. Fortunately for us there is a world in between, where a few dozen stable nuclei are both possible and are actually produced in stars, and are endowed with electron orbitals leading to chemistry with arbitrarily large and complex molecules. This world would disappear with only a few percent fractional change in the quark mass difference in either direction. It does not exist in some closely-related branches of the multiverse wavefunction.

One can estimate roughly the effects of leaving this plane. In that case, nuclear physics is changed in new ways, since the mass of the pion changes. It appears that if the masses 


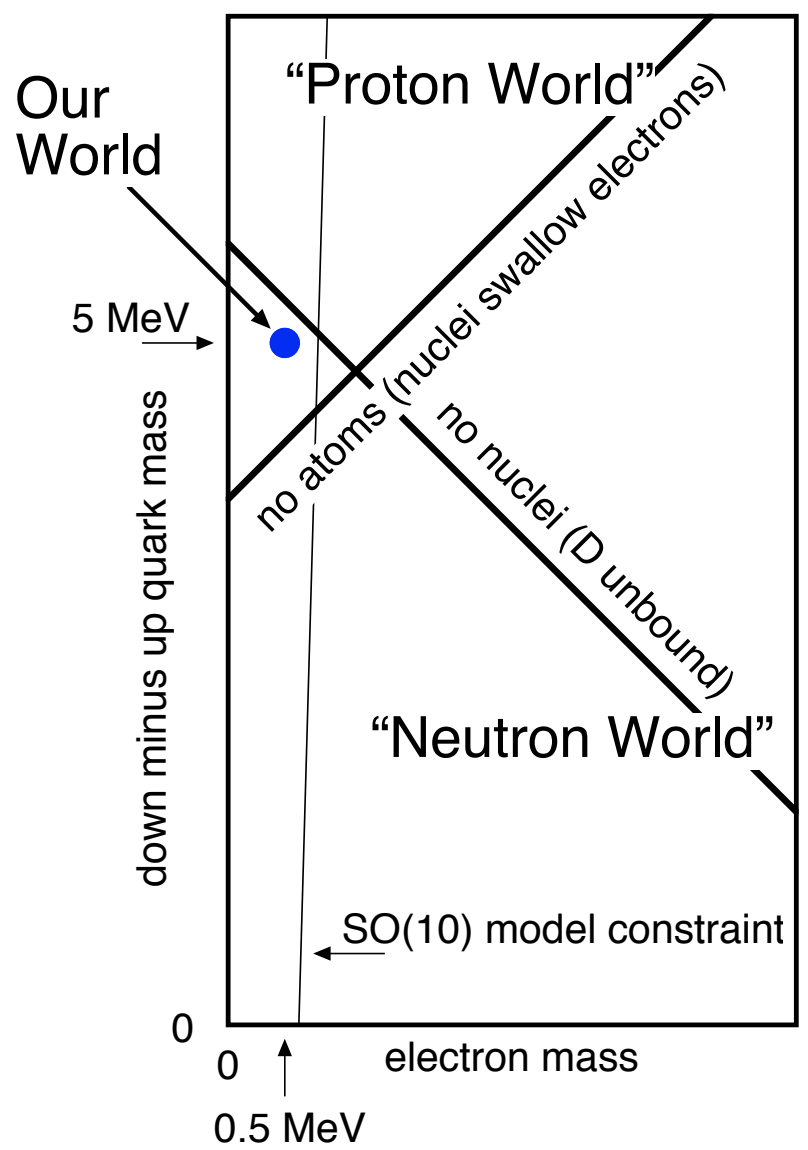

FIG. 1: An overview of simple nuclear physics of the neutron, proton and deuteron, in other universes closely related to ours. Thresholds for various reactions are shown depending on the mass difference between the down and up quark mass, and the electron mass, in the plane where the sum of the up and down masses does not change. Our world is the [pale blue] dot. The SO(10) constraint shown imposes the restriction that the ratio of electron to down quark mass is fixed by a symmetry to have the same value it does in the real world; the region to the right of this is excluded for positive down-quark mass.

are increased by more than about $40 \%$, the range of nuclear forces is reduced to the point where the deuteron is unstable; and if they are reduced by a similar amount, they are strengthened to the point where the diproton is stable. On the other hand, the latter change also reduces the range of nuclear forces so there are fewer stable elements overall. The sum of the quark masses in our world appears roughly optimized for the largest number of stable nuclei. Again, the situation would change qualitatively (e.g., far fewer stable elements) with 
selects a congenial environment (why we do not live on an asteroid or a quasar, etc.) While these seem very different, from a quantum-mechanical perspective they do not differ in kind. Both of them are selections of a congenial branch of the wavefunction of the universe.

In the original formulation of quantum mechanics, it was said that an observation collapsed a wavefunction to one of the eigenstates of the observed quantity. The modern view is that the cosmic wavefunction never collapses, but only appears to collapse from the point of view of observers who are part of the wavefunction. When Schrödinger's cat lives or dies, the branch of the wavefunction with the dead cat also contains observers who are dealing with a dead cat, and the branch with the live cat also contains observers who are petting a live one.

Although this is sometimes called the "Many Worlds" interpretation of quantum mechanics, it is really about having just one world, one wavefunction, obeying the Schrödinger equation: the wavefunction evolves linearly from one time to the next based on its previous state. Anthropic selection in this sense is built into physics at the most basic level of quantum mechanics. Selection of a wavefunction branch is what drives us into circumstances in which we thrive. Viewed from a disinterested perspective outside the universe, it looks like living beings swim like salmon up their favorite branches of the wavefunction, chasing their favorite places.

The selection of a planet or a galaxy is a matter of chance. In quantum mechanics this means a branch of the wavefunction has been selected. The binding energy of our galaxy was determined by an inflaton fluctuation during inflation; that was when the branching occurred that selected the large scale gravitational potential that set the parameters for our local cosmic environment. We can achieve statistical understanding about this kind of selection because we can observe other parts of the ensemble, by observing galaxy clustering, the microwave background, and so on. In this way, we understand the physics of the symmetry breaking. We even know something about the formation of the different galaxy distributions in other universes we will never see. These are regarded as just different by chance.

If the quark and electron masses are also matter of chance, the branching of the wavefunction occurred along with the symmetry breaking that fixed their masses. There may be ways to observe aspects of the statistical ensemble for this event also, by studying the gravitational wave background rather than the microwave background.

We do not know when all the choices of parameters and symmetries are made. Some of 


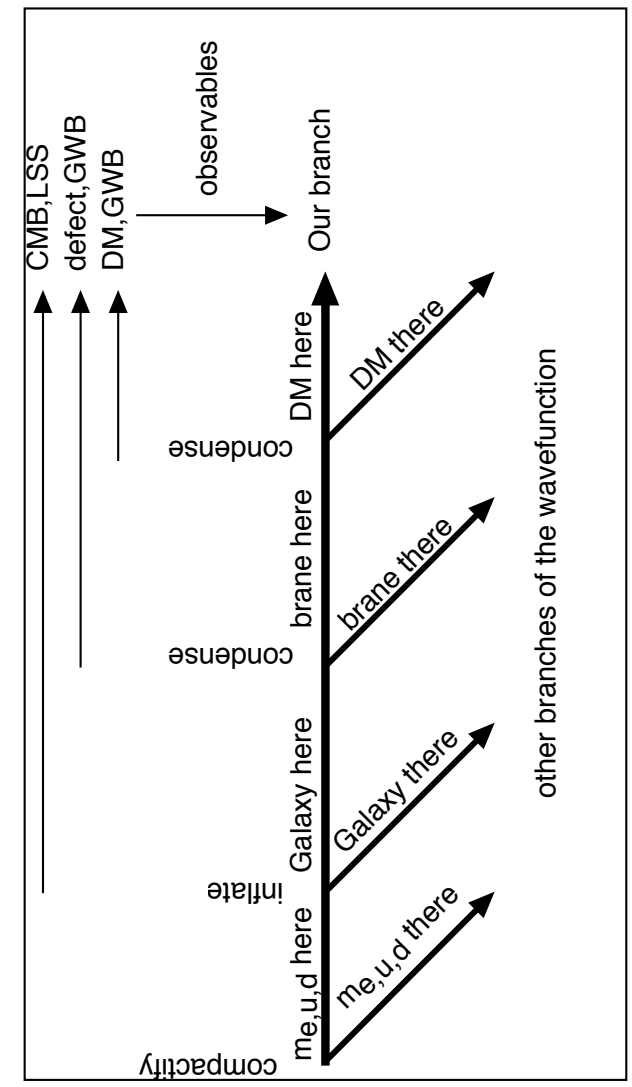

FIG. 3: A schematic sketch of the branching history of the wavefunction to which we belong. At various points in cosmic history, symmetry breaking (e.g., compactification, inflation, condensation) made random choices, which were frozen into features such as standard model parameters, the galaxy distribution, or the dark matter density. In some cases, these events left other observables which can be observed directly in other ways, such as microwave background anisotropy, large scale structure, gravitational wave backgrounds, or cosmic defects. Thus although the other branches of the wavefunction cannot be observed directly, the physics of the branching events in some situations may be independently observable.

these branchings may leave traces of other choices observable in our past light cone. It could be that some parameters are spatially varying even today, in response to spatial variations in scalar or dark matter fields. For example, one model of dark energy predicts large variations in the masses of neutrinos, depending on the local density of the neutrino component of dark matter [10]. (Indeed the basic idea that effective neutrino masses depend on the local physical environment is now part of the standard theory of solar neutrino oscillations.) Thus 
the properties of stars can be spatially modulated depending on the dark matter density - a quantity determined, in many theories, by a branching event that occurred recently enough to have an observable ensemble. Such ideas provide a new motivation for observational programs to quantify the extent to which the constants of nature are really constant in spacetime. (A thriving example of this can be found in studies of varying $\alpha$.)

In some models, events connected with fixing the local quark and electron masses may have happened late enough to leave fossil traces. This could happen during the final compactification of some of the extra dimensions, or the condensation of our own Standard Model 3D brane within a larger dimensional space.

If dimensional compactification happens in a sufficiently catastrophic symmetry breaking, it can lead to a background of gravitational waves. Because they are so penetrating, gravitational waves can carry information directly from almost the edge of our past light cone, well beyond recombination, even beyond weak decoupling - indeed, back to the edge of 3D space as we know it. If the extra dimensions are smaller than the Hubble length at dimensional compactification or brane condensation, their collapse can appear as a first-order phase transition in our 3D space, leading to relativistic flows of mass-energy. If the extra dimensions are larger than or comparable to the Hubble scale, the 3D brane we inhabit may initially condense with warps and wiggles that lead to a gravitational wave background. Either way the mesoscopic, classical motion of branes settling down to their final equilibrium configuration could lead to a strong gravitational-radiation background in a frequency range detectable by detectors now under development 11, 12, 13, 14]. Thus, instruments designed to observe the early boundary of spacetime may also explore the early boundary of physics as we know it, and directly test ideas concerning the separation of various branches of the multiverse having different fundamental parameters.

This blending of empirical cosmology and fundamental physics is reminiscent of our Darwinian understanding of the tree of life. The double helix, the four-base codon alphabet and the triplet genetic code for amino acids, any particular gene for a protein in a particular organism - are all frozen accidents of evolutionary history. It is futile to try to understand or explain these aspects of life, or indeed any relationships in biology, without referring to the way the history of life unfolded. In the same way that (in Dobzhansky's phrase), "nothing in biology makes sense except in the light of evolution", physics in these models only makes sense in the light of cosmology. 


\section{Acknowledgments}

This work was supported by NSF grant AST-0098557 at the University of Washington.

[1] B. Carter, in M. S. Longair (ed.), Confrontation of Cosmological Theory with Observational Data (Reidel, Dordrecht, 1974), p. 291.

[2] B. J. Carr and M. J. Rees, Nature 278, 605 (1979).

[3] R. Bousso and J. Polchinski, "Quantization of four-form fluxes and dynamical neutralization of the cosmological constant," JHEP 0006, 006 (2000) arXiv:hep-th/0004134.

[4] S. Kachru, R. Kallosh, A. Linde and S. P. Trivedi, "De Sitter vacua in string theory," Phys. Rev. D 68, 046005 (2003) arXiv:hep-th/0301240.

[5] L. Susskind, "The anthropic landscape of string theory" arXiv:hep-th/0302219.

[6] C. J. Hogan, "Why the universe is just so," Rev. Mod. Phys. 72, 1149 (2000) arXiv:astro-ph/9909295.

[7] R. N. Cahn, "The eighteen arbitrary parameters of the standard model in your everyday life", Rev. Mod. Phys. 68, 951 (1996)

[8] M. K. Gaillard, P. D. Grannis, and F. J. Sciulli, "The standard model of particle physics", Rev. Mod. Phys. 71, S96 (1999)

[9] E. M. Munro, G. Odell, "Morphogenetic pattern formation during ascidian notochord formation is regulative and highly robust", Development 129, 1 (2002)

[10] R. Fardon, A. E. Nelson and N. Weiner, "Dark energy from mass varying neutrinos," arXiv:astro-ph/0309800.

[11] C. J. Hogan, "Scales of the extra dimensions and their gravitational wave backgrounds," Phys. Rev. D 62, 121302 (2000) arXiv:astro-ph/0009136.

[12] C. J. Hogan, "Gravitational waves from mesoscopic dynamics of the extra dimensions," Phys. Rev. Lett. 85, 2044 (2000) arXiv:astro-ph/0005044.

[13] C. J. Hogan and P. L. Bender, "Estimating stochastic gravitational wave backgrounds with Sagnac calibration," Phys. Rev. D 64, 062002 (2001) arXiv:astro-ph/0104266.

[14] K. Ichiki and K. Nakamura, "Stochastic Gravitational Wave Background in Brane World Cosmology," arXiv:astro-ph/0406606. 